January 2020

\title{
Racial Mislabeling in Multiracial Individuals: Implications for Professional Counseling and Education
}

\author{
C. Peeper McDonald \\ Mercer University \\ Catharina Y. Chang Ph.D. \\ Georgia State University \\ Caroline O'Hara \\ Syracuse University \\ Kan Guvensel \\ Life University \\ Lindy Parker \\ Georgia State University
}

Follow this and additional works at: https://trace.tennessee.edu/tsc

Part of the Counseling Commons, Education Commons, and the Multicultural Psychology Commons

\section{Recommended Citation}

McDonald, C. Peeper; Chang, Catharina Y. Ph.D.; O'Hara, Caroline; Guvensel, Kan; and Parker, Lindy (2020) "Racial Mislabeling in Multiracial Individuals: Implications for Professional Counseling and Education," Teaching and Supervision in Counseling: Vol. 2 : Iss. 1 , Article 2.

https://doi.org/10.7290/tsc020102

Available at: https://trace.tennessee.edu/tsc/vol2/iss1/2

This article is brought to you freely and openly by Volunteer, Open-access, Library-hosted Journals (VOL Journals), published in partnership with The University of Tennessee (UT) University Libraries. This article has been accepted for inclusion in Teaching and Supervision in Counseling by an authorized editor. For more information, please visit https://trace.tennessee.edu/tsc. 


\title{
Racial Mislabeling in Multiracial Individuals: Implications for Professional Counseling and Education
}

\author{
C. Peeper McDonald, Catharina Y. Chang, Caroline O’Hara, Kan Guvensel, Lindy Parker
}

With the extreme growth of people who identify as Biracial or Multiracial, it is evident that their unique racial experiences need to be better understood, especially within the field of professional counseling, to underscore competent practice (Charmaraman, Woo, Quach, \& Erkut, 2014; Jackson, Yoo, Guevarra, \& Harrington, 2012; Tran, Miyake, Martinez-Morales, \& Csizmadia, 2016). As a result, the researchers in this study investigated the lived experiences of racial mislabeling for Multiracial individuals. The participants of this study were $14(\mathrm{n}=14)$ individuals who identified as Multiracial and said they experienced racial mislabeling, a form of racial invalidation. Data were collected through individual, semi-structured interviews. The data was analyzed using phenomenological methods within Moustakas' (1994) transcendental approach, which led to themes that described the essence of the participants' experience. These themes are discussed in detail, and recommendations for researchers and counselor educators to improve training and education are provided.

Keywords: professional counselors, racial mislabeling, counselor education

Williams (1999) discussed the importance of claiming the "I" in her account of growing up in the 1950 s as a Biracial individual in America. She asserted that individuals should choose for themselves how to identify racially and reject the social constructions of race that push individuals into racial boxes. Still, Williams failed to recognize the challenges that individuals face when others reject this alternative classification. For example, Biracial individuals racially classified themselves based on their assumptions of how others perceived their race (Brunsma \& Rockquemore, 2001). Pressure to conform to socially constructed racial categories subverted the empowerment that Williams (1999) spoke of to claim the "I" (Giamo, Schmitt, \& Outten, 2012; Terry \& Winston, 2010).

\section{Multiracial Identity Development Models: Re- search and Andragogy}

Poston (1990) developed a Biracial Identity Development Model that addresses the unique issues of Biracial individuals, but the model fails to account for the effects of being racially misclassified by others. Poston falsely assumed that Biracial individuals' outward appearances match social constructions of race. Cheng and Lee (2009) proposed the Multiracial Identity Integration (MII) concept, which focuses on the negotiation that takes place for Multiracial individuals in their various racial identities. Though MII includes negative and positive racial experiences, the experiences and effects of being categorized as a race that is incongruent with one's identity are left out. More recently, Franco and O'Brien (2018) sought to quantify racial invalidation through the development of a racial identity invalidation scale, helping to close the gap in the literature. However, the majority of research conducted with Biracial individuals is with individuals that have a Black-White racial mix (e.g., Franco, Katz, \& O'Brien, 2016) leaving out individuals with other racial make-ups.

The gaps in Multiracial research form a trickledown effect that has led to a lack of informed training in Multicultural andragogy specifically related to the Multiracial population (Seward, 2014). Though conceptual models exist as tools for counselor education

C. Peeper McDonald, Mercer University; Catharina Y. Chang, Lindy Parker, Georgia State University; Caroline O'Hara, Syracuse University; Kan Guvensel, Life University. Correspondence concerning this article should be addressed to C. Peeper McDonald, mcdonald_cp@mercer.edu 
programs to design and implement multicultural training courses (e.g., Buckley \& Foldy, 2010), there is a lack of information on student of color experiences, including Multiracial students, within counselor education and the implications of this for andragogy (Seward, 2014; Shorter-Gooden, 2009). As a result, educators must examine the curriculum related to the Multiracial population and address the gaps in the literature on Multiracial andragogy.

\section{The Concept of Mislabeling}

Numerous studies explored racial identity development as it relates to counseling competencies and outcomes (i.e., Chao, 2012; Shorter-Gooden, 2009). However, at the time of this study, the authors found no studies examining Multiracial identity development, coupled with the experience of being racially mislabeled. Collins (2000) examined Biracial Japanese American identity development. Participants in this study addressed the importance of being correctly identified racially. Though these researchers examined perceptions of racial identity and how Multiracial individuals assert identity, researchers have yet to explore the assertion or language behind the misperceptions of Multiracial identity. Although Multiracial identity development is a growing area of study, the concept of mislabeling is missing. In response to this gap, mislabeling is the term created by the authors to describe an incident where an individual describes another person's race as something different than how that person self-identifies. The label used to describe the person's race is a mislabel because it is incongruent with how the person identifies. Thus, mislabeling not only speaks to an incorrect assumption one has about a Multiracial person's identity, but mislabeling also describes the verbalization about this assumption of racial identity. The term mislabeler describes the individual doing the mislabeling. The concept that most resembles the construct of mislabeling is racial invalidation under the overarching umbrella of microaggressions, defined as subtle and often unintentional actions that continue to oppress marginalized groups (Sue et al., 2007). Racial invalidation occurs when outside entities pressure the Multiracial person to adopt a singular identity, essentially disregarding the person's Multiracial identity (Franco et al., 2016; Jackson,
Yoo, Guevarra, \& Harrington, 2012). Though mislabeling fits under the general umbrella of these terms, mislabeling is more specific and speaks to the verbal assumptions (via labels) by others about Multiracial identity. This is in contrast to denying one's racial identity, which describes racial invalidation (Franco \& O'Brien, 2018).

\section{Purpose of the Study}

Counselor educators and researchers recommend specific identity-edifying strategies for teaching, supervising, and practicing counseling. Broaching (Day-Vines et al., 2007) helps professional counselors and supervisors learn the identities salient to their clients and supervisees, whereas racial identity development models help address concerns and typical development for Multiracial people. While these strategies help increase our understanding of Multiracial people, we do not know the impact that racial mislabeling has on a person and the complexities that surround the experiences of mislabeling for Multiracial people. To address the gap in the literature, we explored the following research question: What are the experiences of Multiracial individuals who encounter racial mislabeling? By studying mislabeling experiences, the researchers hope to contribute to wellness-based practices taught and utilized by counselor educators and supervisors. Specific areas that could be influenced by the data include Multicultural andragogy and Multicultural counseling techniques and exploration activities taught to counselors in training and used in both the counseling and supervision settings with Multiracial individuals.

\section{Method}

The researchers used a phenomenological qualitative method to describe the meaning of the lived experiences of several Multiracial individuals regarding racial mislabeling (Moustakas, 1994). Phenomenological methodology allows for the points of view of participants to be captured using descriptive, contextual data. Additionally, Moustakas' (1994) transcendental approach was used to couch the data, particularly for bracketing the research teams' values and assumptions of participants' experiences. 


\section{Research Team}

The research team consisted of five individuals. The primary investigator (PI) identified as a Biracial (Native American and White) female while another researcher identified as a White female, and the third researcher identified as a White, international male from Turkey. The remaining two team members served as auditor and advisor to the project and identified as White and Asian, respectively.

\section{Participants}

The researchers used purposive criterion sampling and snowball sampling techniques (Creswell, Hanson, Clark, \& Morales, 2007) to invite individuals who identified as Multiracial, were 18 years or older, and could speak to a specific experience of being racially mislabeled to participate. To specifically underscore snowball sampling, participants were encouraged to refer individuals to the study and pass along study materials to others who fit the inclusion criteria. After receiving Institutional Review Board (IRB) approval, we recruited participants by emailing requests to colleagues and faculty at institutions around the country and by posting on social media (e.g., CESNET electronic mailing list, Facebook). Interested participants were contacted via email by the Principal Investigator (PI) and provided with an IRB-approved consent form. Participants who wanted to continue responded via email to the PI, and phone or in-person interviews were scheduled based on the convenience of the participants.

In total, there were $14(n=14)$ participants. All participants were from across the United States and Canada. Their ages ranged from 19 to 51 years (median age of 31). Eight participants identified as female, five identified as male, and one identified as transgender male. The racial make-up of the participants was diverse: Two identified as Black and White; one identified as Chinese and White; one identified as Black, Puerto Rican, and White; two identified as Black, Native American, and White; three identified as Native American and White; two identified as Hispanic/Latinx and White; one identified as Turkish and Arabic; one identified as Iranian and White; and one identified as Black, Hispanic/Latinx, West Indian, and White.

\section{Research Procedures}

The researchers used Moustakas' (1994) transcendental approach procedures for data collection and analysis. The first procedure, selecting a phenomenon of interest, was based on the PI's personal experiences of being racially mislabeled and the paucity of Multiracial research in general when compared to other groups.

The second procedure, putting aside or bracketing research assumptions and values, was achieved through the intentional, ongoing reflexive discussions by the research team (excluding the project auditor and advisor) about bias throughout the research process. The discussion of our possible presuppositions and interpretations of the participants' experiences created accountability and awareness within the team that ultimately discouraged these biases from coloring the data. It was especially important to ensure our findings reflected participants' experiences and not our own biases, experiences, or values, given the closeness of the topic to the first author (Balkin \& Kleist, 2016).

The third procedure, collecting data directly from individuals who have experienced the phenomenon, was achieved through semi-structured interviews, the transcripts of the interviews, and demographics of the participants (i.e., age, gender identification, race/ethnicity, career/job, and location; Creswell et al., 2007; Groenewald, 2004). Three of the five authors conducted and audiotaped the interviews. The interviews lasted between 40 and 60 minutes. The research team crafted the following interview questions, in order:

Please describe your cultural heritage and how you think others describe you? Please describe your race and how you think others describe your race? How do you define Multiracial? What does being Multiracial mean to you? How do you conceptualize your own racial development? Please describe an experience where you were mislabeled and how did you respond to being mislabeled? Can you describe a time when you were correctly labeled and what was that experience like for you?

Data were collected until we reached saturation, where no new elements of the participants' experiences emerged (Groenewald, 2004). The research 
team transcribed the interviews verbatim to ensure that we captured the essence of the participants' experience. The lead author sent the transcripts via email to participants to member check. None of the participants requested changes to their transcripts.

The fourth and fifth procedures, analyzing data for common themes and describing the essence of participants' experiences, were achieved through horizontalization of the data, whereby the researchers independently listened to the interviews for a sense of "the whole" and then coded statements for units of general meaning (Creswell et al., 2007; Groenewald, 2004). Once this occurred, the researchers independently delineated the units of meaning relevant to the research question, and then met as a team to verify the units of relevant meaning and eliminate redundancies (Groenewald, 2004; Moustakas, 1994). Afterward, clusters of themes were formed by grouping the units of meaning together (Creswell et al., 2007; Groenewald, 2004; Moustakas, 1994). The research team conducted 11 meetings specifically dedicated to the coding process, where each team meeting lasted approximately 1 hour. The research team discussed each code and theme and reached $100 \%$ consensus.

Trustworthiness. Steps were intentionally taken to underscore the study's trustworthiness. The PI kept an audit trail (Creswell et al., 2007). The auditor used the audit trail to review the findings and ensure the conclusions were supported by the data to support confirmability (Creswell et al., 2007). Additionally, member checking was completed again by sending the participants the final themes to review, to ensure the themes accurately represented their mislabeling experiences. None of the participants requested changes or disagreed with the final themes. Other forms of trustworthiness previously mentioned included: Bracketing meetings and ongoing reflection (Groenewald, 2004). Furthermore, dependability was ensured with the use of multiple reviewers to code for emergent themes (Groenewald, 2004; Morrow, 2005).

\section{Results}

Eight major themes emerged. Themes 1-3 represented constructs that couch the phenomenon of mislabeling by giving context to what it means to be
Multiracial, and themes 4-8 represented the specific nature of mislabeling.

\section{Perceptions of Race}

The theme perceptions of race emerged as a way for the participants to explain how they have experienced others trying to understand their Multiracial identity. This theme also emerged as a way for participants to explain that racial identity, for them, was a facet of culture and a socially constructed category that framed their experiences of mislabeling. Others' perceptions of the participants often changed with context and understanding, and led participants to believe that there is no simple definition of race. Participant 11 described personal encounters with people who classified him based on their own understanding of race, without regard for how the participant personally identified: "I've had people insist that racially I have to be White and then I've had people insist that racially I have to be not White based on how they understand what race is." Similarly, Participant 10 reported the many comments he's received about his physical appearance:

Well, a lot of people interestingly enough tell me I look Latin and then some people will tell me I don't. And I've heard somebody tell me I look Mulatto which I'm not sure about and I've heard some people tell me I look more Latin... some people say I have more of a European look... I kind of don't think I look Mexican to be honest, but that might be in my head.

Another example of this theme is seen when Participant 1 reported that the message she often received from others was: "If you're not White skin toned then you're Black." In addition to this binary perception of race, this theme included other concepts, such as: physical features/skin tone are related to race; labeling comes first before interpersonal relations; color and culture can be synonymous terms and sometimes are mutually exclusive; participants reported that location and language affected perception of race (familiarity of race); and participants reported that racial identity is multidimensional. 


\section{Multiracial Categorization}

The theme Multiracial categorization emerged as a result of the participants' perspectives that being categorized as Multiracial included both positive and negative aspects. Participants often described the challenges of navigating the racial identity development process in relation to their mislabeling experiences. Participant 2 explained pressures of representation:

And it kind of bothers me because you are seen as a representative of a certain culture or religion, then you have the pressure of behaving a certain way because you know even though you are an individual and it's impossible for one person to represent the whole culture. But you know people see you that way. Then you kind of filter the things that you want to say, the behaviors that you want to do.

This theme can be further described using these concepts participants reported: the universality of the minority experience; Multiracial people are often asked to identify their race in everyday conversation; experiencing stereotypes related to race; being Multiracial is developmental and fluid; Multiracial does not look one way; being Multiracial is a unique experience that monoracial people cannot understand; Multiracial identity should be celebrated and it means "the best of both worlds." Additionally, belongingness appeared so frequently within the data that it warranted a designation as a subtheme given that feelings of belongingness contributed to the participants' understanding of their Multiracial categorization. This subtheme had a binary aspect, where participants discussed times when their sense of belongingness was underscored by their family and culture. At the same time, belongingness was affected by participants' family or culture and led them to feel removed or isolated. For example, Participant 13 discussed a time in college where her Multiracial categorization was highlighted by a sense of connectedness to others:

My undergraduate time actually being the first time I had people of color openly saying to me these kinds of things and realizing, "wow, there are other people out there who have gone through something like what I've gone through." However, Participant 1 spoke to the lack of feelings of belongingness and acceptance with others: "I feel, to this day, I still feel that the only place that I truly belong is at home with my family... (others) just don't think I belong at all." Belongingness can be further described using concepts where being Multiracial meant the following: connection to numerous cultures and not feeling a part of any one culture; being affected by nuclear family influence; experiences of racism and discrimination.

\section{Turning Point in Racial Development}

The theme turning point in racial development emerged from the data as a way to describe major developmental events that contributed to the participants' experience and understanding of Multiracial identity development that framed their understanding of mislabeling experiences. Participant 12 described a vivid early childhood memory:

One of the key points I remember from being a child is not wanting to be Hispanic at all. I think I remember going to sleep and before going to bed I would do my prayers... and I would always slip in something where I wish I would wake up White.

Participant 7 reported that a turning point in racial development was the experience of being treated differently by different groups in her extended family: "On my mother's side, the Caucasian side of my family, they did not accept me at all... So growing up, not understanding at such a young age why."

The data from this theme set the stage for participants' future mislabeling experiences. This theme can be further described using concepts where participants reported the following: beliefs that minority people have an "inner-knowing" that signals to them that "they're different from an early age"; beliefs that lighter skin and the categorization of White was more desirable; searching out diverse experiences within diverse communities as a way to become more racially aware.

\section{Context of Mislabeling}

The theme context of mislabeling emerged as a way to describe when, where, and by whom racial mislabeling occurred. Participants revealed that racial mislabeling can happen anywhere and any time, and be perpetrated by anyone. Participant 5 described 
the experience of being mislabeled by her middle school peers on the bus:

This one dude was like, "you know we have to sit in the back and you have to sit in the front," and I was like, "who is he talking to?" Like, does he think that I'm White, because I'm not, and at the time I didn't know anything about what race was until I got into college and I'm like, "I'm not White, just because I have light skin don't mean that I'm White."

Similarly, Participant 4 reported, "I think another place that I am most often mistaken for something else... is in the dating world." This theme can be further described using the following concepts that emerged from the data: mislabeling occurred frequently; mislabeling can occur in almost every known setting (i.e., school, police, place of employment, counseling workshops); and mislabeling was based on appearance.

\section{Impacts of Mislabeling}

The theme impacts of mislabeling emerged as a way to describe how the participants responded to being mislabeled and the emotional ramifications (subthemes of interpersonal and intrapersonal ramifications) of this experience. The interpersonal ramifications described the effects of being mislabeled between the participant and others. For example, Participant 14 described an interpersonal ramification of mislabeling:

Well mostly some of my mislabeling has been around racial slurs... where I work... some of the women who worked there would only speak Spanish to me, fully knowing that I didn't speak Spanish, I mean that's just racist. In that context I didn't stay at the job... I couldn't effectively do my job, so I gave my two weeks' notice, finished out and left.

Interpersonal ramifications can be further described using the following concepts: mislabeling created a lack of connection between the mislabeler and the mislabeled; language barriers contributed to mislabeling; the mislabeled often used humor and treated race lightly to diffuse the mislabel; mislabeling can occur with anyone; mislabeling made the mislabeled feel "othered" or not enough; and mislabeling felt like an attack from the mislabeler. Intrapersonal ram- ifications included the internal impact that being mislabeled had on the participant. In response to being mislabeled as solely White, Participant 6 reported "So it just hurts. It angers me to a certain extent." Intrapersonal ramifications can be further described using the following concepts: mislabeling caused anger, frustration, and disappointment; mislabeling contributed to feelings of being objectified and judged as a person; the mislabeled desired to be heard regarding mislabeling experiences and desired to be seen as a person first rather than identified by group membership.

\section{Correcting the Mislabel}

The theme correcting the mislabel emerged from the data as a way to describe the action on the participants' part when racial mislabeling took place and included subthemes related to interpersonal and intrapersonal ramifications. Participants reported going through a process of decision making when faced with a mislabeler. Participants sometimes chose to correct the mislabeling and inform the mislabeler of the mistake. Other times, participants chose not to correct. The concept of correction was complex because it involved the process of decision making in choosing to correct or not correct and the interpersonal and intrapersonal ramifications that participants considered before moving forward with either choice. Participant 13 shared that the choice to correct was based on the perceived assessment of the mislabeler's responsiveness or apparent ignorance, speaking to the subtheme of interpersonal ramifications: "I only talk to people who I believe are going to be responsive. If I think people are on a certain level of ignorance I might have to deal with that too." The internal impact of choosing to correct (or not) is described in the subtheme intrapersonal ramifications, as seen in Participant 3's quote:

I don't mind correcting racial or cultural identity confusion, I don't mind correcting family of origin mislabeling, but if the very core of my being is challenged for having the inner views and things that I hold and it is deep within me, then I will just let it be mislabeled and misunderstood.

This theme can be further described using the following concepts related to correction: did not occur 
when deep emotions were involved; was an emotional investment for participants; varied with context; occurred if the mislabeler appeared open and approachable; did not occur if the situation did not appear safe; was exhausting and tiring.

\section{Being Correctly Labeled}

The theme being correctly labeled emerged as a result of adding an interview question early on in the interview process as the antithesis to the experiences of being mislabeled. "Can you describe a time when you were correctly labeled and what was that experience like for you?" was added to the interview questions in response to an experience the PI had in interviewing a participant who correctly identified the interviewer. An example of this can be seen when Participant 11 described her surprise at being correctly labeled by a fellow peer: "She just correctly identified me so I know my reaction to that was almost on the opposite side, surprise, like 'Wow, no one's ever you know gotten that right before." Participant 9 described the impact of being correctly labeled: "It makes me feel safe. It makes me feel like I can have a good conversation with them, and I feel like they're more intelligent." This theme can be described further using the following concepts that emerged from the data regarding being correctly labeled: led to positive emotions and a positive experience of the person who correctly labeled; led to feelings of validation; often occurred by a person who shared the same racial make-up as the participant; rarely occurred.

\section{Resistance and Resilience}

The theme resistance and resilience emerged as a way to describe the reactions, thoughts, and behaviors of the participants as forms of "fighting back" and staying strong in the face of mislabeling experiences. This theme exemplified individuals in a reframed way, in which they were not victims, but rather, were reacting normally to an abnormal and unjust situation. This theme differs from that of correcting the mislabel because this theme described an overall state of being that encouraged the participants to act as agents of change in their lives, whereas the correcting the mislabel theme described the in-themoment decision-making process (and the ramifica- tions thereof) that occurred when mislabeling happened. The data from the resistance and resilience theme described participants behaving in ways that resisted the system of mislabeling while protecting themselves. An example of this can be seen in the desire of Participant 11 to challenge others' presuppositions about identity while being mindful of the risks: "That is a way to share knowledge so I'm happy to do it, I'm just trying to go about it in a safe way." Furthermore, Participant 3 described her ongoing journey of resilience and resistance:

My thought process has very much served me, it mimics Dr. King in which he said he refused to take offense, he refused to allow someone to make him feel any different than the way that he felt... so I think it's an increased intentionality or mindfulness of being heard versus being angry or offended or hurt.

This theme can further be described using the following concepts that emerged from the data related to participants' experiences: desired to break stereotypes and be inclusive of minority voices; learned how to navigate multiple cultural and "different worlds"; wanted to be heard regarding mislabeling experiences; saw themselves as multiple cultures that made one unified identity; confronted mislabeling and assumptions head on.

\section{Discussion}

We explored Multiracial individuals' experiences of mislabeling, a specific type of microinvalidation. Using transcendental phenomenology (Moustakas, 1994), eight major themes emerged from the data with implications for counseling practice, training, and research. Overall, participants revealed the general oversimplification of the construct of race and that racial mislabeling happened in multiple settings. Given the paucity of literature on this issue and the potential for mislabeling to occur in both the classroom and clinical settings, the invisibility of the Multiracial community continues to perpetuate. As a result, we recommend the following strategies to counteract this phenomenon within the counselor education and supervision settings. 


\section{Counselor Practice and Training Implications}

The findings related to the theme perceptions of race have major implications for our society and especially for the practice of counseling and training. Due to the growing racial milieu of U.S. society (Charmaraman, Woo, Quach, \& Erkut, 2014), it is important for those living in the United States to understand that race is not as simple as skin tone, as it is a complex social construction (Franco \& O'Brien, 2018; Tran et al., 2016). It is especially important for professional counselors, educators, and supervisors working from a foundation of wellness (Kaplan, Tarvydas, \& Gladding, 2014) to build upon this foundation utilizing an understanding of the social construction of race (Giamo et al., 2012; Terry \& Winston, 2010). Specifically, professional counselors, educators, and supervisors should focus on increasing their awareness of the detrimental effects that racial mislabeling can have on the relationship between clients and professional counselors, students and educators, and supervisees and supervisors (Ratts, Singh, Nassar-McMillan, Butler, \& McCullough, 2015).

The theme turning point in racial development highlighted results found in a study by Cheng and Lee (2009), who proposed the MII construct that focuses on the negotiation that takes place for Multiracial individuals in their different identities. For the participants in this study, negotiation of racial identities was an ongoing aspect of development and formed the basis of racial understanding and important turning points throughout their life. For professional counselors, educators, and supervisors, it is important to create space to process these turning points as contributors to past and current negotiations of identity as a way to seek understanding (Ratts et al., 2015).

Correcting the mislabel speaks to previous findings that the process of correcting the mislabel, similar to Collins' (2000) description of positive assertion of identity, is an emotional and conflictual process affecting the individual's relationship with the mislabeler and the individual internally. Though Collins' findings emerged from Biracial Japanese American participants, the process of correcting another's incorrect assumptions by asserting one's identity is similar across multiple mixtures of racial identity. This supports the need to incorporate the correction process into counseling practice and training. Furthermore, counselor educators should revisit racial identity models given that current models still often assume that Multiracial identity is congruent with how others' perceive the Multiracial person (e.g., Poston, 1990). A discussion around racial microaggressions and the effects mislabeling can have on Multiracial individuals within both the supervisory alliance and the therapeutic alliance is warranted.

\section{Counseling Research Implications}

The theme context of mislabeling underscores the idea that society promotes mixed racial messages. Racial mislabeling occurs in a variety of settings with a variety of people (Schlabach, 2013; Terry \& Winston, 2010). Though professional counselors, educators, and supervisors may be inclined to believe that racial mislabeling occurs outside academia and the wellness perspective, the data revealed that they often miss the mark and contribute to racial stereotypes and mislabeling due to lack of knowledge and exposure to Multiracial people. It is increasingly important for professional counselors, educators, and supervisors to recognize that we have not surpassed the necessity for continued work and reflection on race and its impact on society, clients, students, and supervisees. There is more social justice and advocacy work to be done regarding race and race relations, and this should be the guiding research force.

The theme impacts of mislabeling supported previous knowledge of detrimental effects related to being a Multiracial individual (Franco et al., 2016; Giamo et al., 2012). The current study adds to this body of literature by speaking to the detrimental effects relating to both interpersonal and intrapersonal impacts that also accompany the experience of being mislabeled, a specific type of spoken racial invalidation. This theme underscores the need for the counseling profession to recognize that there is more work to be done beyond simply understanding the experiences of a Multiracial individual. This work should focus on the labeling that occurs with these individuals and seek to change this categorization system to one that encourages the label to come from the individual (client, student, or supervisee), or for there to be room for no label at all, based on the individual's preference (Day-Vines et al., 2007; Williams, 1999). 
The empowerment and choice involved in this fit within the constructs of the wellness, social justice, and advocacy foundations of professional counselors, counselor educators, and supervisors (Ratts et al., 2015).

The theme being correctly labeled emerged as a result of an addendum to the interview questions that occurred after an interview where a participant correctly labeled the PI. The researchers felt it was necessary to ask not only about experiences of mislabeling but to explore times when experiences of correct labeling occurred. Though the overwhelming majority of Multiracial literature focuses on detrimental implications of Multiracial identity (Franco et al., 2016; Giamo et al., 2012; Jackson et al., 2012), progress is being made where some researchers are focusing on positive outcomes of Multiracial identity (Binning, Unzueta, Huo, \& Molina, 2009). Additionally, though Remedios and Chasteen (2013) hypothesized about the importance of being accurately identified, the experience of being correctly labeled and positive outcomes that result constitute a completely new area of research.

The theme resistance and resilience underscores the wellness foundation of counseling that fosters well-being and growth for all (Kaplan et al., 2014). The participants' ability to do this took the form of resisting social constructs of what a Multiracial person looks and acts like while demonstrating resolve to make choices around correction and self-identity. The participants often used their experiences as a platform for personal advocacy and encouraging self-identification (Williams, 1999) while integrating this process as a way of life, much like Poston's (1990) description of the integration stage of Biracial identity development. This adds to the minimal literature available on positive aspects of Multiracial identity experiences (Binning et al., 2009) and encourages further study of positive outcomes that could inform counseling work, education, supervision, and social justice and advocacy efforts.

\section{Limitations and Future Directions}

One limitation of the study is the potentially leading questions that some phenomenological experts might argue goes against the goal of pure phenomenology. The use of additional probing questions could have potentially limited the information given by the participants and led them to discuss only specific elements regarding their experiences of being mislabeled. The use of these questions was by design because the research question was not to get at the broad experience of being a Multiracial individual, but was designed to arrive at the essence of the experience of being racially mislabeled while identifying as a Multiracial person. Nonetheless, any factor that could potentially limit the quality of responses given by participants pertaining to the essence of their mislabeling experiences should be considered a limitation.

Another limitation is how individuals who identify as Biracial are subsumed into the same Multiracial category. The rationale for including these individuals in the same racial category were twofold: (a) based on other literature (i.e., Franco \& O'Brien, 2018; Tran et al., 2016), and (b) so as not to exclude either group from expressing their experiences of racial mislabeling. Nonetheless, this could send the message that these groups are synonymous when some individuals may define Biracial to mean a person who identifies with two races while Multiracial could indicate identification with three or more races (Renn, 2000). Though these groups share a nonmonoracial identity, the intention is not to inadvertently mislabel the participants by using the broad term of Multiracial.

The themes expressed in this study serve as a starting point for others to understand racial classification biases and encourage professional counselors, educators, and supervisors to create a safe space that empowers clients, students, and supervisees to express their racial identity. Future research should focus on understanding these themes and using these to inform multicultural ethical standards to better serve this population. This can be done by focusing the scope of Multiracial research. One area in need of research is the experience of racial mislabeling within the transgender community. One of the participants spoke about his gender transition being a major turning point that could not be separated from racial development. Though this was a unique experience, the current researchers believe this to be a meaningful intersectionality of identities that warrants further research. Another area of future research should investigate the role age plays in Multiracial identification and meaning. It appeared that the older participants who were also not affiliated with counseling-related 
Racial Mislabeling

fields had a unique perspective of racial identity that was not present in any of the other participants' interviews. For example, these participants were able to discuss experiences of racial mislabeling but were quick to discuss the "superficiality" of race or the idea that "race shouldn't matter." This seemed almost counter to the ideas of other participants that racial identification and correction of mislabels played a huge part in their own meaning-making and racial development. Though some researchers examined the racial color-blind attitudes of Multiracial individuals and the relationship to Multiracial identity (McDonald, Chang, Dispenza, \& O'Hara 2019), more research is needed to explore the unique racial perceptions and experiences of Multiracial people.

The interpretation of results allows us to provide suggestions for work with clients, students, and supervisees that echo the work of Deters (1997). First, professional counselors, educators, and supervisors should acknowledge that race is a social, fluid, and developmental construction. Additionally, professional counselors, educators, and supervisors should underscore social justice and advocacy initiatives and work to understand how external and internal forces can lead to oppression, discrimination, and stereotyping related to racial identity (Ratts et al., 2015). Thirdly, professional counselors, educators, and supervisors need to support racial ambiguity by working to not have to label others by describing oneself in labels that support who one is as a racial and cultural being and invite others to share what is comfortable for them, accepting the labels they choose to use or not use. Finally, professional counselors, educators, and supervisors need to ask rather than assume someone's racial identification (or any other identification) by consistently practicing multicultural broaching as a way to build rapport and wellness within the relationship (Day-Vines et al., 2007). As the data revealed, verbalizing assumptions about identity could have negative effects on the relationship while forcing the person to choose whether or not to correct the mislabel and how to proceed. As a result, it is imperative for professional counselors and educators to acknowledge the power and responsibility they have and to invite the client, student, or supervisee into the process of coconstructing and meaning-making of identity.

\section{Conclusion}

Understanding experiences of Multiracial persons regarding mislabeling, a specific kind of microaggression, is paramount for multicultural competence for professional counselors and counselor educators. The purpose of this study was to examine these mislabeling experiences and the context of these experiences. The following themes emerged: perceptions of race; Multiracial categorization; milestones in racial development; context of mislabeling; impacts of mislabeling; correcting the mislabel; being correctly labeled; and resistance and resilience. Professional counselors, educators, and supervisors can use these themes to better understand experiences of Multiracial people, determine how to effectively work with and broach multicultural identities, and ultimately underscore multicultural competence.

\section{References}

Balkin, R., \& Kleist, D. (2016). Counseling research: A practitionerscholar approach. Alexandria, VA: American Counseling Association.

Binning, K. R., Unzueta, M. M., Huo, Y. J., \& Molina, L. E. (2009). The interpretation of Multiracial status and its relation to social engagement and psychological well-being. Journal of Social Issues, 65(1), 35-49. doi:10.1111/j.1540-4560.2008.01586.x

Brunsma, D. L., \& Rockquemore, K. (2001). The new color complex: Appearances and Biracial identity. Identity, 1(3), 225-246. doi: 10.1207/S1532706XID0103 03

Buckley, T. R., \& Foldy, E. G. (2010). A pedagogical model for increasing race-related multicultural counseling competency. The Counseling Psychologist, 38, 691-713. doi:10.1177/0011000009360917

Chao, R. (2012). Racial/ethnic identity, gender-role attitudes, and multicultural counseling competence: The role of multicultural counseling training. Journal of Counseling \& Development, 90(1), 35-44. doi: 10.1002/j.1556-6676.2013.00082.x

Charmaraman, L., Woo, M., Quach, A., \& Erkut, S. (2014). How have researchers studied Multiracial populations? A content and methodological review of 20 years of research. Cultural Diversity and Ethnic Minority Psychology, 20(3), 336-352. doi: 10.1037/a 0035437

Cheng, C., \& Lee, F. (2009). Multiracial identity integration: Perceptions of conflict and distance among Multiracial individuals. Journal of Social Issues, 65, 51-68. doi:10.1111/j.15404560.2008.01587.x

Collins, J. (2000). Biracial Japanese American identity: An evolving process. Cultural Diversity \& Ethnic Minority Psychology, 6(2), 115-133. doi:10.1037//1099-9809.6.2.115

Creswell, J. W., Hanson, W. E., Clark, V. L. P., \& Morales, A. (2007). Qualitative research designs: Selection and implementation. The Counseling Psychologist, 35, 236-264. doi:10.1177/0011000006287390

Day-Vines, N. L., Wood, S. M., Grothaus, T., Craigen, L., Holman, A., Dotson-Blake, K., \& Douglass, M. J. (2007). Broaching the 
subjects of race, ethnicity, and culture during the counseling process. Journal of Counseling \& Development, 85, 401-409. doi:10.1002/j.1556-6678.2007.tb00608.x

Deters, K. A. (1997). Belonging nowhere and everywhere: Multiracial identity development. Bulletin of the Menninger Clinic, 61(3), 368. Retrieved from https://search. ebscohost.com/login.aspx?direct=true \&AuthType $=\mathrm{ip}$, shib $\& \mathrm{db}=$ slh $\& \mathrm{AN}=9708133322 \&$ ite $=$ ed slive \&scope $=$ site \&custid $=$ mer 1

Franco, M. G., Katz, R., \& O’Brien, K. M. (2016). Forbidden identities: A qualitative examination of racial identity invalidation for Black/White Biracial individuals. International Journal of Intercultural Relations, 50, 96-109. doi:10.1016/j. ijintrel.2015.12.004

Franco, M. G., \& O’Brien, K. M. (2018). Racial identity invalidation with Multiracial individuals: An instrument development study. Cultural Diversity and Ethnic Minority Psychology, 24(1), 112-125. doi:10.1037/cdp0000170

Giamo, L. S., Schmitt, M. T., \& Outten, H. R. (2012). Perceived discrimination, group identification, and life satisfaction among Multiracial people: A test of the rejection-identification model. Cultural Diversity and Ethnic Minority Psychology, 18(4), 319328. doi:10.1037/a0029729

Groenewald, T. (2004). A phenomenological research design illustrated. International Journal of Qualitative Methods, 3(1), 42-55. doi:10.1177/160940690400300104

Jackson, K. F., Yoo, H., Guevarra, R. J., \& Harrington, B. A. (2012). Role of identity integration on the relationship between perceived racial discrimination and psychological adjustment of Multiracial people. Journal of Counseling Psychology, 59(2), 240-250. doi: $10.1037 / \mathrm{a} 00$

Kaplan, D. M., Tarvydas, V. M., \& Gladding, S. T. (2014). 20/20: A vision for the future of counseling: The new consensus definition of counseling. Journal of Counseling \& Development, 92(3), 366-372. doi:10.1002/j.1556-6676.2014.00164.x

McDonald, C. P., Chang, C., Dispenza, F., \& O’Hara, C. (2019). The relationships among Multiracial identity, color-blind racial ideology, and discrimination in Multiracial individuals: Implications for professional counseling and counselor education. Journal of Counseling \& Development, 97, 75-85. doi: 10.1002/jcad.12237

Morrow, S. L. (2005). Quality and trustworthiness in qualitative research in counseling psychology. Journal of Counseling Psychology, 52(2), 250-260. doi:10.1037/00220167.52.2.250

Moustakas, C. E. (1994). Phenomenological research methods. Thousand Oaks, CA: SAGE.
Poston, W. (1990). The Biracial identity development model: A needed addition. Journal of Counseling \& Development, 69(2), 152-155. doi: 10.1002/j.1556-6676.1990.tb01477.x

Ratts, M. J., Singh, A. A., Nassar-McMillan, S., Butler, S. K., \& McCullough, J. R. (2015). Multicultural and social justice counseling competencies. Alexandria, VA: American Counseling Association.

Remedios, J. D., \& Chasteen, A. L. (2013). Finally, someone who "gets" me! Multiracial people value others' accuracy about their race. Cultural Diversity and Ethnic Minority Psychology, 19(4), 453-460. doi:10.1037/a0032249

Renn, K. A. (2000). Patterns of situational identity among Biracial and Multiracial college students. Review of Higher Education, 23(4), 399-420. doi: 10.1353/rhe.2000.0019

Schlabach, S. (2013). The importance of family, race, and gender for Multiracial adolescent well-being. Family Relations, 62(1), 154174. doi:10.1111/j.1741-3729.2012.00758.x

Seward, D. X. (2014). Multicultural course pedagogy: Experiences of master's-level students of color. Counselor Education and Supervision, 53(1), 62-79. doi: 10.1002/j.15566978.2014.00049.x

Shorter-Gooden, K. (2009). Teaching diversity to the oppressed: Understanding and engaging students of color. In J. L. Chin (Ed.), Diversity in mind and in action (Vol. 2, pp. 129-143). Westport, CT: Greenwood Press.

Sue, D. W., Capodilupo, C. M., Torino, G. C., Bucceri, J. M., Holder, A. B., Nadal, K. L., \& Esquilin, M. (2007). Racial microaggressions in everyday life: Implications for clinical practice. American Psychologist, 62(4), 271-286. doi: 10.1037/0003066X.62.4.271

Terry, R. R., \& Winston, C. E. (2010). Personality characteristic adaptations: Multiracial adolescents' patterns of racial self-identification change. Journal of Research on Adolescence, 20(2), 432455. doi:10.1111/j.1532-7795.2010.00638.x

Tran, A. G. T. T., Miyake, E. R., Martinez-Morales, V., \& Csizmadia, A. (2016). "What are you?" Multiracial individuals' responses to racial identification inquiries. Cultural Diversity and Ethnic Minority Psychology, 22(1), 26-37. doi: 10.1037/cdp0000031

Williams, C. (1999). Claiming a Biracial identity: Resisting social constructions of race and culture. Journal of Counseling \& Development, 77(1), 32-35. doi: 10.1002/j.15566676.1999.tb02412.x 